\title{
Neural Network Analysis for RF to DC signal conversion circuit
}

\author{
Varindra Kumar \\ Department of Engineering \\ Imperial College London \\ London, UK
}

\begin{abstract}
Voltage amplification is an important element of the energy harvesting process to provide an enhanced voltage and run various digital circuits at low power. At RF frequencies, the discrete circuit arrangement using high frequency diode HSMS 2850 and capacitors have been used here to provide the voltage amplification with high power efficiency. The paper describes a mechanism of the Dickson circuit arrangement with its various parameters. The power efficiency is obtained and compared with multiple stages of the Dickson configuration for the input power, frequency of operation and its load using keysight based ADS tool. Finally a neural network has been designed using a Matlab script and the trained neural network has been applied to get the efficiency result. The result shows that with an increase in the multiplier stage of the Dickson circuit, there is a significant increase in its power efficiency. In addition the neural network implementation shows that the circuit parameters can be obtained using a well-trained neural network script.
\end{abstract}

Keywords-Schottky Diode; Energy harvesting; Dickson; Voltage doubler

\section{INTRODUCTION}

Recent years have seen an increase in miniaturized electronics IoT devices with multiple I/O interfaces running the audio, video and data storage applications in wireless and wired network. These electronics devices have traditionally been powered using bulky power regulators with batteries and its associated circuitries. Replacing the battery is often cumbersome and requires extra resource and human intervention. This replacement becomes specifically difficult when the devices are deployed in harsh and hazardous and out of human reach environment. At the same time, once these batteries become depleted, these electronic devices become useless even causing a hazard. One potential option is to have batteryless operation or a hybrid battery solution with a selfpower generating source. The recent breakthroughs in the schottky diode technology within RF and high frequency range has contributed to harvest the power from various external energy sources and convert it to an appreciable DC voltage. The external energy sources may arise from the piezo based vibration, temperature gradient, solar power, movement, light, wind power, electromagnetic source [1] etc. Many diode configuration circuits have been modeled for the efficiency calculation in the past, however to my knowledge the neural network analysis has not been performed. The purpose of this paper is to design and present various stages of Dickson voltage multiplier and provide a comparative value of simulation results for its power efficiency for the application of input power and operating frequency. During the dynamic load operation, the load resistance often changes based on the current requirement of its load, hence the power efficiency of the load variation from a nominal load of $100 \Omega$ to high load of $12 \mathrm{k} \Omega$ has also been calculated here. The paper also presents a design of the neural network to calculate the efficiency of multiplier stage based on the calculation of the result of onestage Dickson voltage doubler circuit. The error of the neural network derived output response and actual output has been obtained and its performance and regression plot has also been obtained to show the neural network efficacy.

\section{Design Methodology}

The cascade arrangement of RF diodes with high switching speed and low voltage diode drop can provide a rectification mechanism for the low voltage input, creating a charge pump circuit. Although there have been mentioned many configurations in literatures [2], the Dickson circuit arrangement for its high energy efficiency has been used here. The Dickson voltage multiplier circuit for the stage - one to stage - eight are shown in Figs 1 - 4. Each stage of the circuit consists of two diode and capacitors arrangement, while the output of the n-stage voltage multiplier provides the output. Here ' $n$ ' indicates the number of stage multiplication, $V_{o}$ is the open circuit voltage of single stage, $R_{o}$ is the internal resistance and $R_{L}$ is the value of the load resistor. The cascade arrangement of the capacitor and diode arrangement provide a passive voltage offset before its rectification. The $\mathrm{C} 1$ and D1 pair provides a rectification of the peak value while the D2 and C2 pair forms a circuit for its voltage pump. Here the obtained output voltage while ignoring the diode threshold voltage is approximately twice the applied input voltage, and hence the circuit can be described as a voltage doubler circuit and can produce $2 n$ times the input voltage using (1) [3]. Similarly if the capacitance is assumed to be short circuit, the diodes create an antiparallel circuit and hence the input impedance of the circuit can be calculated using (2) while the output power and efficiency can be obtained using (3) and (4) [4 - 5]. 


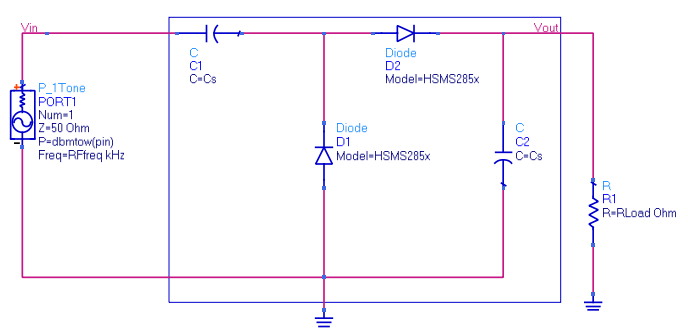

Fig. 1. Dickson $x 1$ configuration

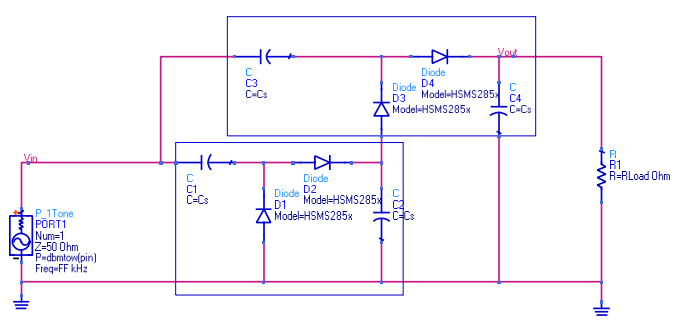

Fig. 2. Dickson $\mathrm{x} 2$ configuration

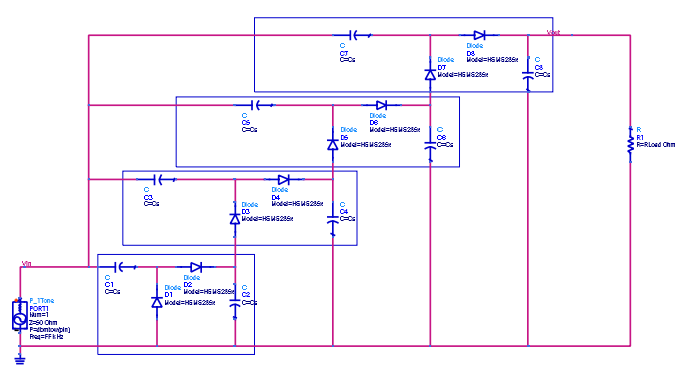

Fig. 3. Dickson $\mathrm{x} 4$ configuration

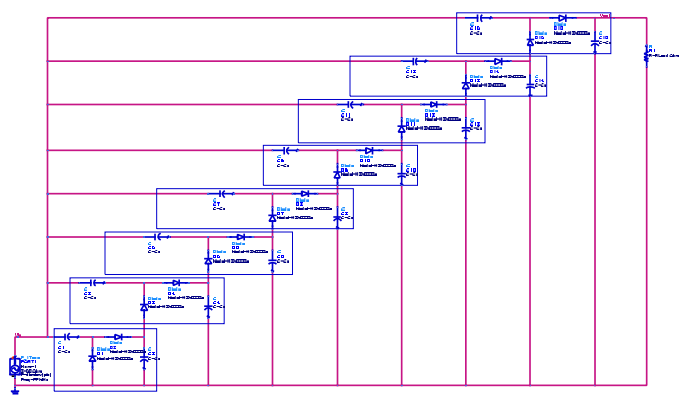

Fig. 4. Dickson $\mathrm{x} 8$ configuration

$$
\begin{gathered}
V_{\text {out }}=n\left(V_{p}-V_{D}\right) \\
Z_{\text {in }}=\frac{Z_{D}}{n} \\
P_{\text {out }}=\frac{V_{\text {out }}^{2}}{R_{L}} \\
\eta_{\mathrm{C}}=\frac{P_{\text {out }}}{P_{\text {in }}^{\text {inc }}-P_{\text {in }}^{\text {ref }}}
\end{gathered}
$$

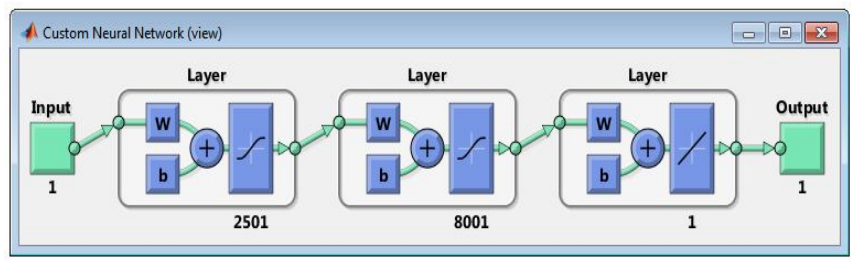

Fig. 5. Neural Network configuration

So far little research has been done on the application of the artificial neural network (ANN) for the calculation of electrical parameters such as efficiency, a typical neural network used in this application is shown in Fig. 5. The network used here is feed forward network with multilayer perceptron for training the input. Here the input takes the parametric input of the stage 1 circuit while three intrinsic layers have been trained to produce the output. The weight and threshold of the function is adjusted till the error of the difference in output is within a considerable confidence interval. The classic gradient descent function using 'traingd' and trainlm with 'LavenbergMarquardt' function has been used to train the system to derive its output [6 - 9]. The neural network GUI tool has been deactivated while a small neural network script has been written to train the system. The input is passed through three layers of network with layer one and two consisting of 2501 and 8001 neurons using tansig functions for its activation to obtain the error margin of 1e-3 while layer three consists of one number of neuron with a linear function. The plot of the tansig and linear function as defined using (5) and (6) is obtained and shown in Fig. 6. Here the learning rate is defined as $1 \mathrm{e}-2$.

$$
\tan \operatorname{sig}(x)=\frac{2}{[1+\exp (-2 x)]}
$$

$$
\operatorname{purelin}(x)=x
$$

\section{Tansig(x) function Linear(x) function}
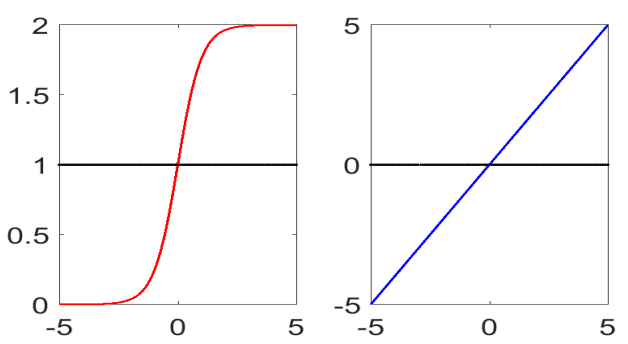

Fig. 6. Tansig and Purelin function for the neural network

\section{Modelling And Simulation Results}

The ADS (Advanced Designed System) software from Keysight has been used to model and simulate the diode configuration. Here the spice model of the Agilent schottky diode HSMS 2850 [10-11] and $90 \mathrm{pF}$ capacitors are used for the modeling of various multiplication stages. The input power with $\mathrm{P}_{-}$1Tone function, load resistance and frequency are varied using Harmonic Balance (HB) and the effect of the output power is obtained using ADS tool and compared with various stages of the amplification. These plots have been obtained using Python, an open source tool. 


\section{A. Spice results for the configuration}

Fig. 7 shows the obtained efficiency when the input power is swept from the $-10 \mathrm{dBm}$ to $50 \mathrm{dBm}$ with an increment of 1 $\mathrm{dBm}$. As seen here, the stage one provides the least efficiency with a maximum of $5 \%$ of all these four different stages while the stage eight provides the highest efficiency with the efficiency reaching upto $72 \%$.

\section{Dickson Efficiency (\%) plot with power}

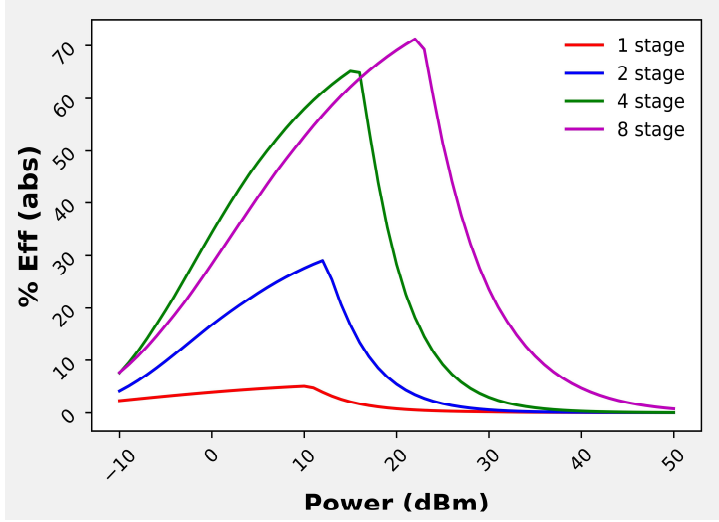

Fig. 7. Efficiency plot with input power

\section{Dickson Efficiency (\%) plot with freq}

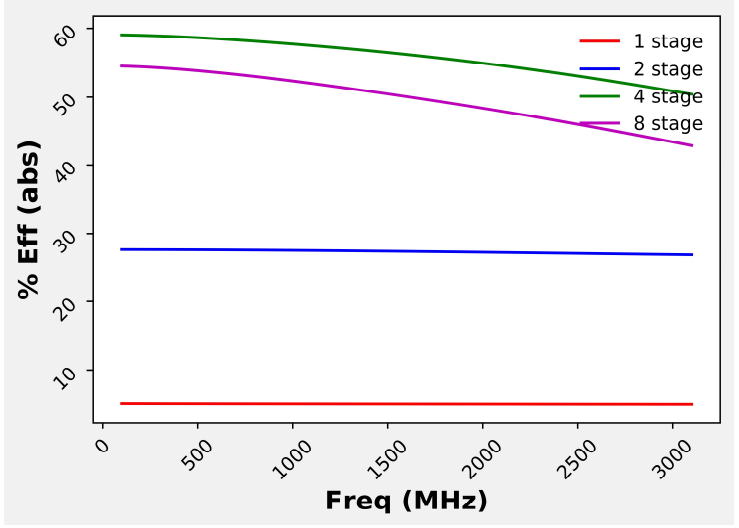

Fig. 8. Efficiency plot with operating frequency

Dickson Efficiency (\%) plot with RLoad

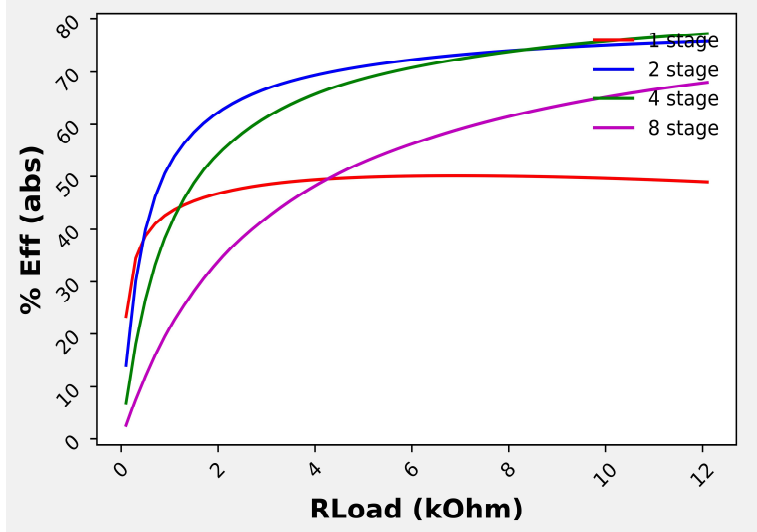

Fig. 9. Efficiency plot with resistive load
At the same time, the higher stage requires higher input power for the circuit to operate beyond the threshold voltage of the diode circuit arrangement. Fig. 8 shows the obtained power efficiency when the frequency is varied from $100 \mathrm{MHz}$ to 3.1 $\mathrm{GHz}$ with an increment of $100 \mathrm{MHz}$. Here the stage one circuit provides the least efficiency with $5 \%$ efficiency across the entire frequency spectrum while the stage four circuit provides the highest efficiency with $60 \%$. Fig. 9 shows the power efficiency behavior with a sweep in the resistive load from 100 to $12 \mathrm{k} \Omega$. The stage two and four circuit shows a similar behavior with highest efficiency of about $75 \%$.

\section{B. Neural Network results for the configuration}

Once the power efficiency plot for one stage circuit is obtained using the ADS tool, the neural network script is run to obtain the efficiency plot of various stages for its variation in input power, frequency and load resistance. As seen from Fig. 10, the obtained efficiency plot for the stage two with respect to the applied input power is quite comparable to the result obtained through ADS tool.

\section{Dickson config Efficiency (\%) with Input power}

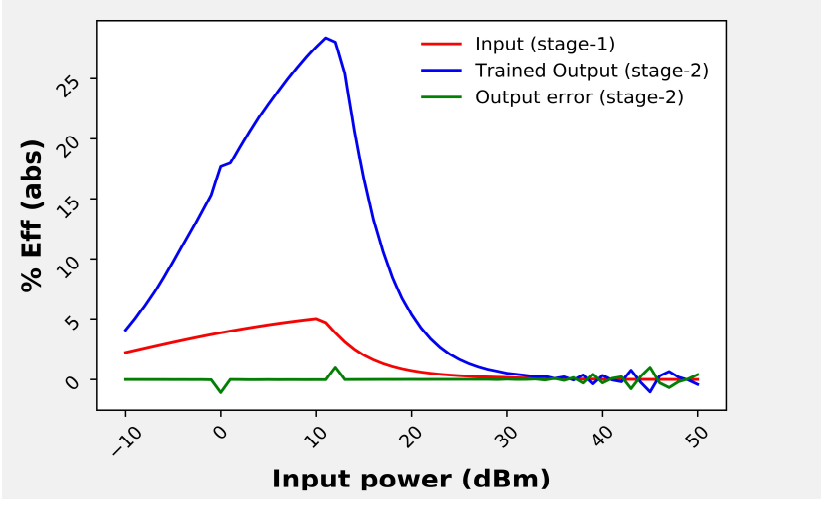

Fig. 10. Stage 2 Efficiency plot with input power using neural network

\section{Dickson config Efficiency (\%) with Input power}

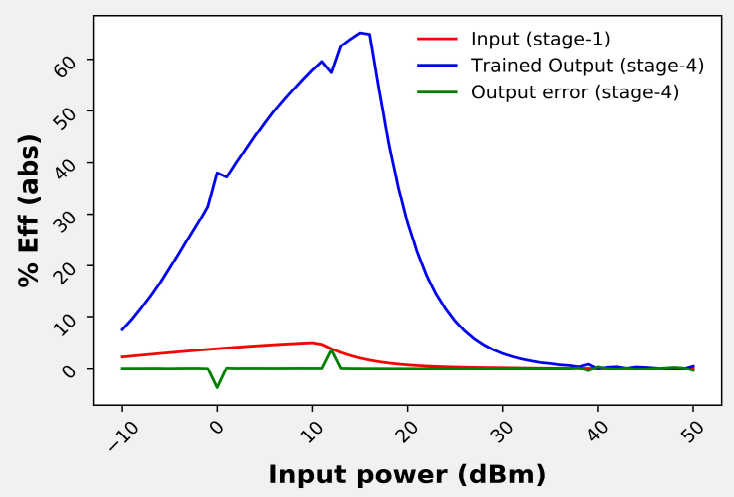

Fig. 11. Stage 4 Efficiency plot with input power using Nneural network

Here the error between the actual output of the ADS tool to the neural network trained output is also shown and is considerably small. Similarly Figs. $11-12$ show the trained power efficiency output and the error with the actual output for the stage 4 and stage 8 . Figs. $13-15$ show the output 
power efficiency plot and the error with the actual output power efficiency for the stage two, four and eight with a variation in frequency using the trained neural network that has been described in the previous section.

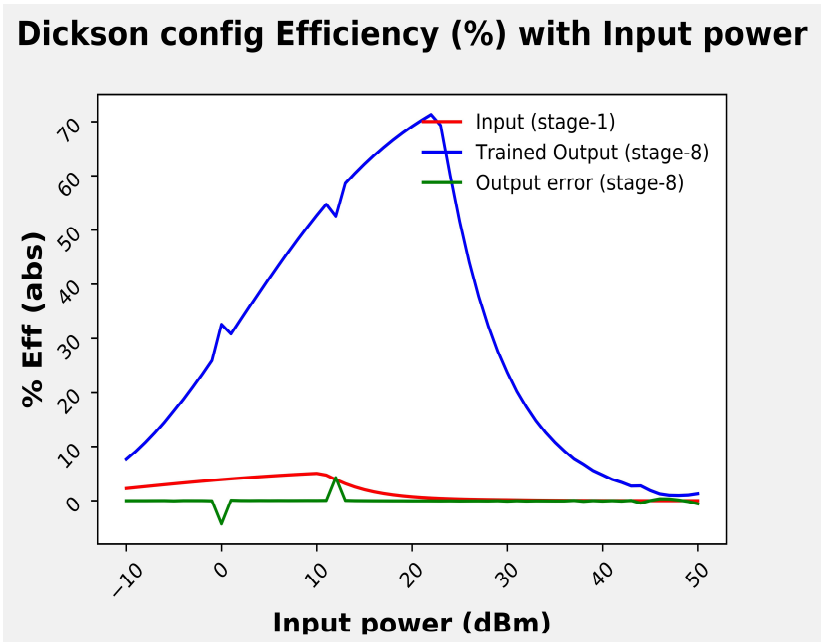

Fig. 12. Stage 8 Efficiency plot with input power using neural network

Dickson config Efficiency (\%) with freq

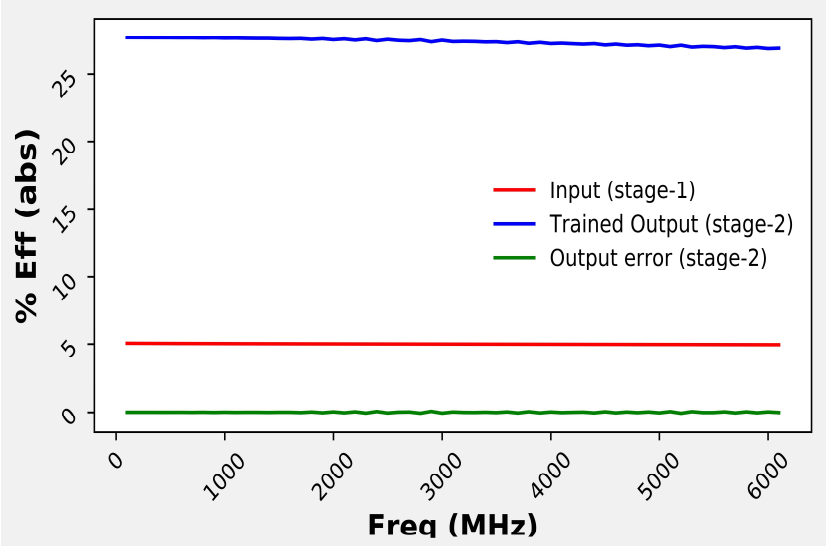

Fig. 13. Stage 2 Efficiency plot with frequency using neural network

Dickson config Efficiency (\%) with freq

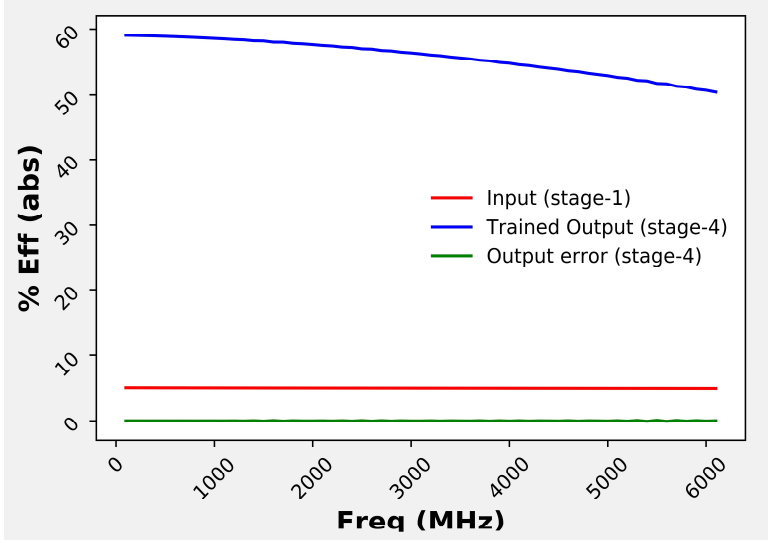

Fig. 14. Stage 4 Efficiency plot with frequency using neural network
Dickson config Efficiency (\%) with freq

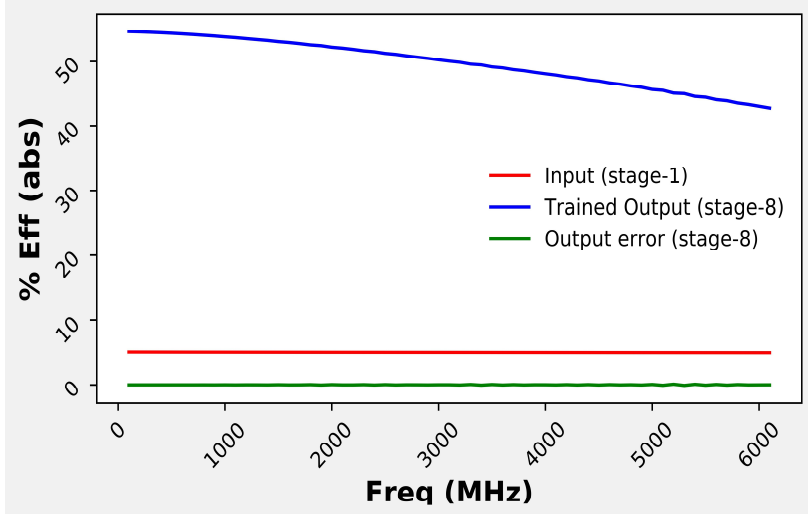

Fig. 15. Stage 8 Efficiency plot with frequency using neural network

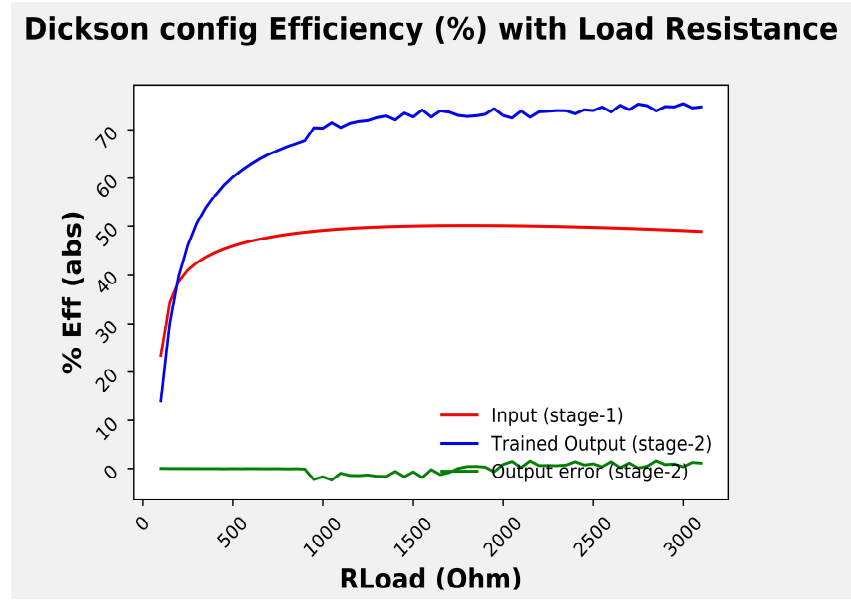

Fig. 16. Stage 2 Efficiency plot with load resistance using neural network

\section{Dickson config Efficiency (\%) with Load Resistance}

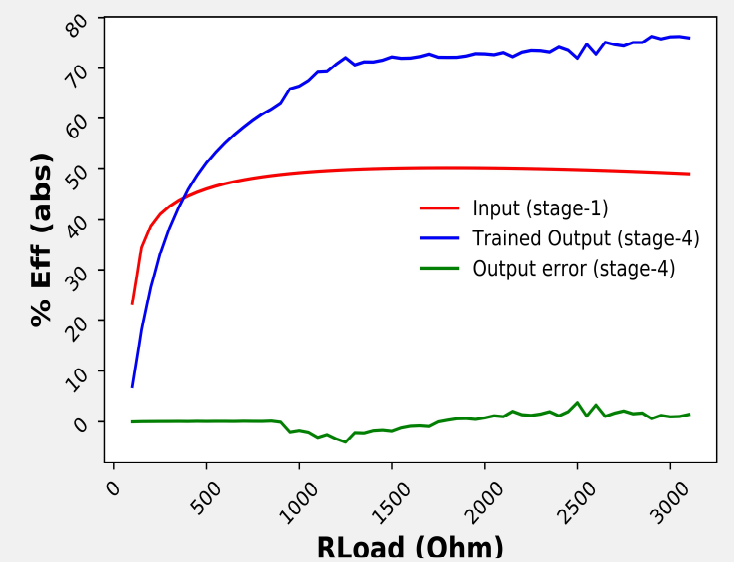

Fig. 17. Stage 4 Efficiency plot with load resistance using neural network

Here the trained neural network output shows the same behavior as its original circuit response. Similarly Figs. $16-$ 18 show the output power efficiency plot and the error with the actual output power efficiency for the stage two, four and eight with a variation in the load resistance using the trained neural network as described in the previous section. The error 
between the actual output and neural net trained output shows the error margin within $1 \%$. This shows that the trained neural network can provide multi-parametric results and can be used in predicting the multi-stage efficiency.

\section{Dickson config Efficiency (\%) with Load Resistance}

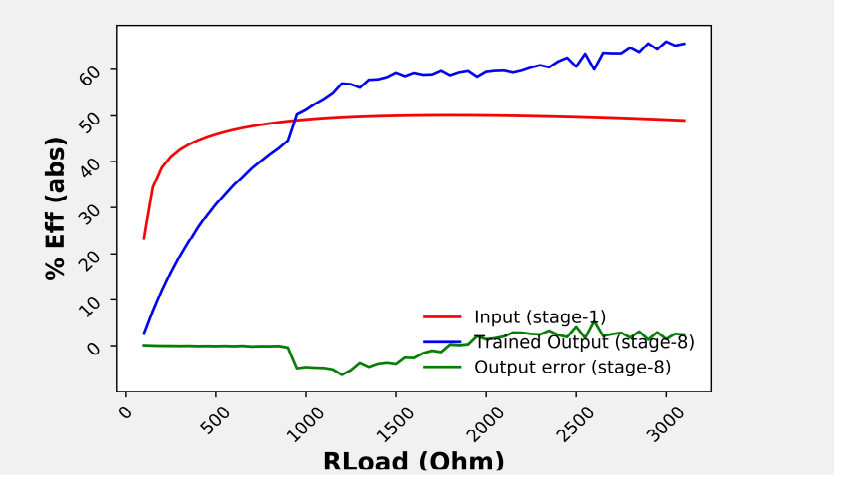

Fig. 18. Stage 8 Efficiency plot with load resistance using neural network

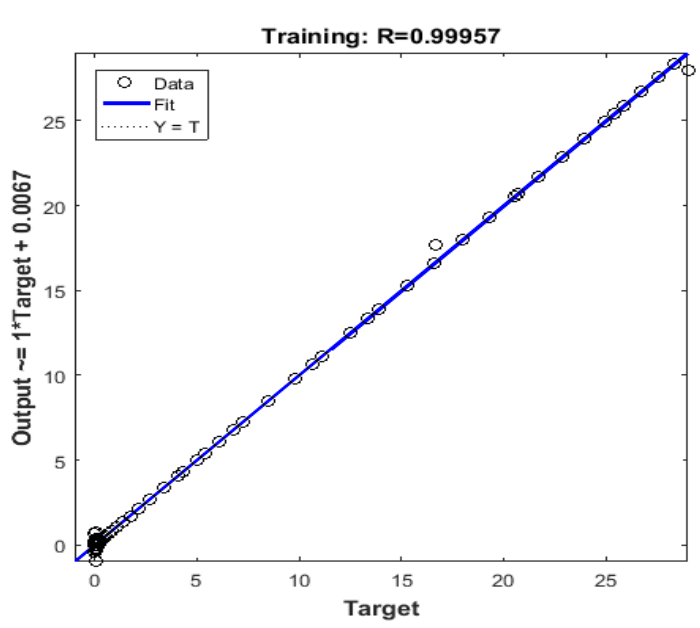

Fig. 19. Plot regression for the stage 2 efficiency

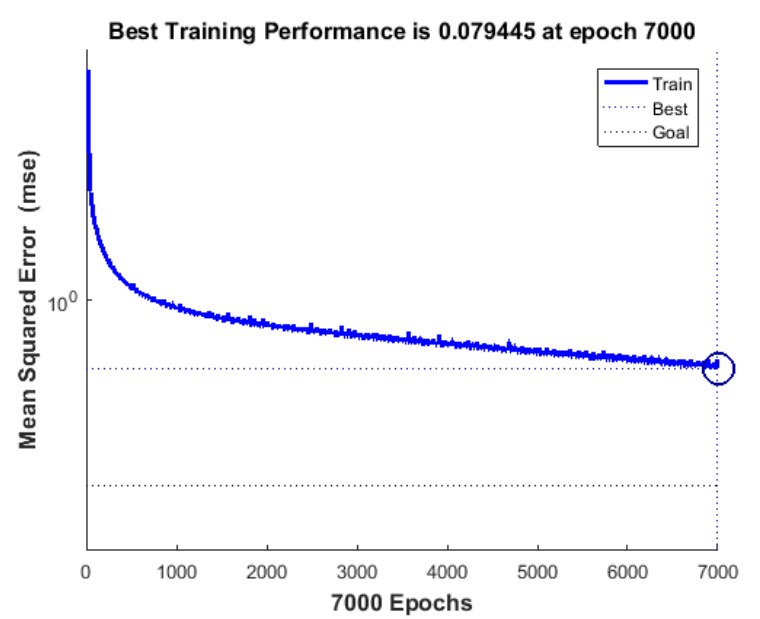

Fig. 20. Plot performance for the stage 2 efficiency

The regression and performance plot for the power efficiency with respect to the input power, operating frequency and load resistance for various stages has been obtained however for its clarity and conciseness only the result of the stage two circuit has been plotted and shown in Figs. $19-20$. As shown in Fig. 19 for its linear regression plot, the neural network obtained data shows the best fit for its output result.

\section{CONCLUSION}

The RF devices are widely used across communication systems and it requires careful planning of an efficient power delivery method to its circuitry. The suggested work provides a mechanism to increase the efficiency of power delivery devices till an appreciable value of $70 \%$ using discrete components with an increase in multiplier stage circuit, providing a better power efficiency at low input voltage. A neural network has been trained to calculate the efficiency. Various obtained results within a very small margin to its original value show that the neural network can provide a viable option for the calculation of various parameters instead of relying on spice tools.

\section{ACKNOWLEDGMENT}

The author would like to thank the Department of Aeronautics Engineering, Imperial College London for providing the necessary support and access for the project.

\section{REFERENCES}

[1] M. M. Tentzeris and Y. Kawahara, "Novel Energy Harvesting Technologies for ICT Applications," 2008 International Symposium on Applications and the Internet, Turku, 2008, pp. 373-376, doi: 10.1109/SAINT.2008.113

[2] J. F. Dickson, "On-chip high-voltage generation in MNOS integrated circuits using an improved voltage multiplier technique," in IEEE Journal of Solid-State Circuits, vol. 11, no. 3, pp. 374-378, June 1976, doi: $10.1109 /$ JSSC.1976.1050739

[3] A. Sedra and K. Smith, Microelectronic Circuits, 5th ed., Oxford University Press: Oxford, UK, 2004, pp. 189-190.

[4] J. Curty, N. Joehl, C. Dehollain and M. J. Declercq, "Remotely powered addressable UHF RFID integrated system," in IEEE Journal of SolidState Circuits, vol. 40, no. 11, pp. 2193-2202, Nov. 2005, doi: 10.1109/JSSC.2005.857352

[5] F. Yuan, CMOS circuits for passive wireless microsytems, Springer, 2011.

[6] Christopher M. Bishop, Pattern Recognition and Machine Learning, Springer, 2006.

[7] J. Arif, N. Ray Chaudhuri, S. Ray and B. Chaudhuri, "Online Levenberg-Marquardt algorithm for neural network based estimation and control of power systems," 2009 International Joint Conference on Neural Networks, Atlanta, GA, 2009, pp. 199-206, doi: 10.1109/IJCNN.2009.5179071

[8] Y.-C. Du and A. Stephanus, "Levenberg-Marquardt Neural Network Algorithm for Degree of Arteriovenous Fistula Stenosis Classification Using a Dual Optical Photoplethysmography Sensor," Sensors 2018, 18, 2322. doi: $10.3390 / \mathrm{s} 18072322$

[9] J. A. R. Argota and I. Anitzine, "ANN-based model for predicting RF signal received power in indoor propagation links," DEStech Transactions on computer science and engineering, 2017. doi:10.12783/dtcse/mcsse2016/11001

[10] Avago Technologies HSMS-285x Series Surface Mount Zero Bias Schottky Detector Diodes. Available online: https://docs.broadcom.com/doc/AV02-1377EN (accessed on 5 October 2020).

[11] R. R. Buted, Zero Bias Detector Diodes for the RF/ID Market, Hewlett Pack. J. 1995, 46, 94. 